\title{
Ruthenium-catalyzed Decarboxylative Allylation of Nonstabilized Ketone Enolates.
}

\author{
Erin C. Burger and Jon A. Tunge* \\ Department of Chemistry, University of Kansas, Lawrence, KS 66045
}

\section{Supporting Information}

\section{Experimental}

Materials. Methylene chloride and tetrahydrofuran were dried over activated alumina. ${ }^{1}$ All other materials were used as received. $\beta$-ketoesters $\mathbf{1 a - 1} \mathbf{j}$ were prepared by DMAPcatalyzed addition of diketene to the corresponding allylic alcohols. ${ }^{2} \quad \beta$-ketoesters 4 and 6a were prepared by the DMAP catalyzed condensation of $\beta$-ketoethylesters and allylic alcohols. ${ }^{3}{ }^{1}$ H NMR spectra were obtained on a Bruker Avance 400 or a Bruker Avance 500 DRX spectrometer and referenced to residual protio solvent signals. Structural assignments are based on ${ }^{1} \mathrm{H},{ }^{13} \mathrm{C}$, DEPT-135, COSY, and HMQC spectroscopies.

Catalyst Screening: An NMR tube was charged with 1a $(20 \mathrm{mg})$. The appropriate solvent, catalyst, and ligand were added. ${ }^{1} \mathrm{H}$ NMR spectra were recorded after 1.5, 4, and $16 \mathrm{~h}$ and the conversions determined by integration. Branched to linear ratios were determined by comparison of the ${ }^{1} \mathrm{H}$ NMR spectra with those of $\mathbf{2 a}$ (vide infra) and $\mathbf{3 a} \mathbf{a}^{4}$

General procedure for catalytic Carroll rearrangements: In a Schlenk tube under argon, $[\mathrm{Cp} * \mathrm{RuCl}]_{4}(2.5 \mathrm{~mol} \%)$ and bipyridine $(10 \mathrm{~mol} \%)$ were dissolved in $2 \mathrm{~mL}$ of methylene chloride. The resulting deep purple solution was allowed to stir briefly before addition of allyl- $\beta$-ketoester $(1 \mathrm{mmol})$ in $3 \mathrm{~mL}$ of methylene chloride via cannula. The reaction was allowed to stir under Ar until the resulting dark burnt orange solution returned to purple. Following solvent evaporation the crude product was purified via flash chromatography $\left(\mathrm{SiO}_{2}, 15 \% \mathrm{Et}_{2} \mathrm{O}\right.$ : Hex $)$, providing the products in $>95 \%$ purity as determined by ${ }^{1} \mathrm{H}$ NMR spectroscopy.

\footnotetext{
${ }^{1}$ Pnagborn, A. B.; Giardello, M. A.; Grubbs, R. H; Rosen, R. K.; Timmers, F. J. Organometallics 1996, $15,1518-20$.

${ }^{2}$ Collado, I.; Pedregal, C.; Mazon, A.; Espinosa, J. F.; Blanco-Urgoiti, J.; Schoepp, D. D.; Wright, R. A.; Johnson, B. G.; Kingston, A. E. J. Med.Chem. 2002, 45, 3619-3629.

${ }^{3}$ Gilbert, J. C.; Kelly, T. A. J. Org. Chem. 1998, 63, 449-450.

${ }^{4}$ Oi, S.; Honma, Y,; Inoue, Y. Org. Lett. 2002, 4, $667-669$.
} 


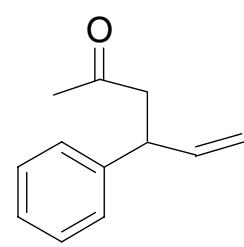

2a: ${ }^{5}$ pale yellow oil : ${ }^{1} \mathrm{H}$ NMR $\left(400 \mathrm{MHz}, \mathrm{CDCl}_{3}\right) \delta 7.28(\mathrm{~m}, 5 \mathrm{H}$ : arom $\mathrm{H}), 5.99$ (ddd, $J=$ $\left.7 \mathrm{~Hz}, 11 \mathrm{~Hz}, 17 \mathrm{~Hz}, 1 \mathrm{H}: \mathrm{CH}=\mathrm{CH}_{2}\right), 5.08\left(\mathrm{~d}, J=11 \mathrm{~Hz}, 1 \mathrm{H}: \mathrm{CH}=\mathrm{CH}(H)_{\mathrm{E}}\right), 5.04(\mathrm{~d}, J=$ $\left.17 \mathrm{~Hz}, 1 \mathrm{H}: \mathrm{CH}=\mathrm{CH}(H)_{\mathrm{z}}\right) 3.94$ (app. q, $J=7 \mathrm{~Hz}, 1 \mathrm{H}: \mathrm{CH}$ ), $2.91(\mathrm{dd}, J=7 \mathrm{~Hz}, 16 \mathrm{~Hz}, 1 \mathrm{H}$ : diastereotopic $\left.\mathrm{CH}_{2}\right) 2.84\left(\mathrm{dd}, J=7 \mathrm{~Hz}, 16 \mathrm{~Hz}, 1 \mathrm{H}\right.$ : diastereotopic $\left.\mathrm{CH}_{2}\right) 2.12$ (s, $3 \mathrm{H}$ :

$\mathrm{CH}_{3}$ ). ${ }^{13} \mathrm{C}$ NMR $\left(75 \mathrm{MHz}, \mathrm{CDCl}_{3}\right) \delta 207.50(\mathrm{C}=\mathrm{O}), 143.19$ (arom. C), 140.94 $\left(\mathrm{CH}=\mathrm{CH}_{2}\right), 129.04$ (arom. $\mathrm{CH}$ ), 128.01 (arom. $\mathrm{CH}$ ), 127.03 (arom. $\mathrm{CH}$ ), 115.04 $\left(\mathrm{CH}=\mathrm{CH}_{2}\right), 49.40\left(\mathrm{CH}_{2}\right), 44.95(\mathrm{CH}), 31.10\left(\mathrm{CH}_{3}\right) . \mathrm{IR}\left(\mathrm{CH}_{2} \mathrm{Cl}_{2}\right): v_{\max } 1716,1157,994$, 922. HRMS calcd for $\mathrm{C}_{12} \mathrm{H}_{13} \mathrm{O}[\mathrm{M}-\mathrm{H}]$ 173.0966, found 173.0954.

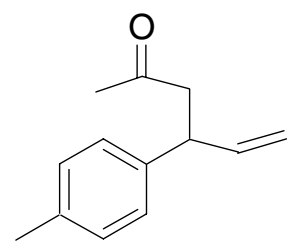

2b: pale yellow oil: ${ }^{1} \mathrm{H}$ NMR (400 MHz, $\left.\mathrm{CDCl}_{3}\right) \delta 7.13(\mathrm{~m}, 4 \mathrm{H}$ : arom $\mathrm{H}$ ), 5.97 (ddd, $J=$ $\left.7 \mathrm{~Hz}, 10 \mathrm{~Hz}, 17 \mathrm{~Hz}, 1 \mathrm{H}: \mathrm{CH}=\mathrm{CH}_{2}\right), 5.07\left(\mathrm{~d}, J=10 \mathrm{~Hz}, 1 \mathrm{H}: \mathrm{CH}=\mathrm{CH}(H)_{\mathrm{cis}}\right), 5.03(\mathrm{~d}, J=$ $\left.17 \mathrm{~Hz}, 1 \mathrm{H}: \mathrm{CH}=\mathrm{CH}(H)_{\text {trans }}\right) 3.90$ (app. q, $\left.J=7 \mathrm{~Hz}, 1 \mathrm{H}: \mathrm{CH}\right), 2.89$ (dd, $J=7 \mathrm{~Hz}, 16 \mathrm{~Hz}$, 1H: diastereotopic $\left.\mathrm{CH}_{2}\right) 2.83\left(\mathrm{dd}, J=7 \mathrm{~Hz}, 16 \mathrm{~Hz}, 1 \mathrm{H}\right.$ : diastereotopic $\left.\mathrm{CH}_{2}\right) 2.34$ (s, $3 \mathrm{H}$ : $\left.\mathrm{Ph}-\mathrm{CH}_{3}\right) 2.11$ (s, 3H: $\left.\mathrm{CH}_{3}\right) .{ }^{13} \mathrm{C}$ NMR $\left(75 \mathrm{MHz}, \mathrm{CDCl}_{3}\right) \delta 207.63(\mathrm{C}=\mathrm{O}), 141.18$ $\left(\mathrm{CH}=\mathrm{CH}_{2}\right), 140.161$ (arom. C), 136.57 (arom. C), 129.73 (arom. $\mathrm{CH}$ ), 127.68 (arom. $\mathrm{CH}), 114.81\left(\mathrm{CH}=\mathrm{CH}_{2}\right), 49.48\left(\mathrm{CH}_{2}\right), 44.62(\mathrm{CH}), 31.09\left(\mathrm{CH}_{3}\right) 21.43\left(\mathrm{Ph}-\mathrm{CH}_{3}\right)$. IR $\left(\mathrm{CH}_{2} \mathrm{Cl}_{2}\right): v_{\max } 1716,1352,1157$. HRMS calcd for $\mathrm{C}_{13} \mathrm{H}_{17} \mathrm{O}[\mathrm{M}+\mathrm{H}] 189.1279$, found 189.1278 .

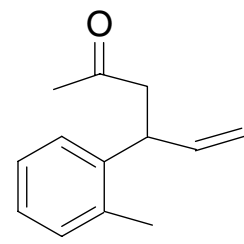

2c: pale yellow oil: ${ }^{1} \mathrm{H}$ NMR $\left(400 \mathrm{MHz}, \mathrm{CDCl}_{3}\right) \delta 7.16(\mathrm{~m}, 4 \mathrm{H}$ : arom $\mathrm{H}), 5.93$ (ddd, $J=$ $\left.7 \mathrm{~Hz}, 10.3 \mathrm{~Hz}, 17 \mathrm{~Hz}, 1 \mathrm{H}: \mathrm{CH}=\mathrm{CH}_{2}\right), 5.06\left(\mathrm{~d}, J=10.3 \mathrm{~Hz}, 1 \mathrm{H}: \mathrm{CH}=\mathrm{CH}(H)_{\mathrm{cis}}\right), 4.96(\mathrm{~d}, J$ $\left.=17 \mathrm{~Hz}, 1 \mathrm{H}: \mathrm{CH}=\mathrm{CH}(H)_{\text {trans }}\right) 4.19$ (app. q, $\left.J=7 \mathrm{~Hz}, 1 \mathrm{H}: \mathrm{CH}\right), 2.93(\mathrm{dd}, J=7 \mathrm{~Hz}, 16.5$ $\mathrm{Hz}, 1 \mathrm{H}$ : diastereotopic $\left.\mathrm{CH}_{2}\right) 2.85\left(\mathrm{dd}, J=7 \mathrm{~Hz}, 16.5 \mathrm{~Hz}, 1 \mathrm{H}\right.$ : diastereotopic $\left.\mathrm{CH}_{2}\right) 2.40$ (s, 3H: $\left.\mathrm{Ph}-\mathrm{CH}_{3}\right) 2.14$ (s, $\left.3 \mathrm{H}: \mathrm{CH}_{3}\right) .{ }^{13} \mathrm{C}$ NMR $\left(75 \mathrm{MHz}, \mathrm{CDCl}_{3}\right) \delta 207.50(\mathrm{C}=\mathrm{O}), 141.15$ (arom. C), $140.56\left(\mathrm{CH}=\mathrm{CH}_{2}\right), 136.40$ (arom. C), 131.04 (arom. $\left.\mathrm{CH}\right), 126.80$ (arom. $\mathrm{CH}$ ),

\footnotetext{
${ }^{5}$ Snider, B. B.; Patricia, J. J. J. Org. Chem. 1989, 54, 38-46.
} 
126.76 (arom. CH), 126.62 (arom. $\mathrm{CH}) 114.97\left(\mathrm{CH}=\mathrm{CH}_{2}\right), 48.84\left(\mathrm{CH}_{2}\right), 40.21(\mathrm{CH})$, $31.05\left(\mathrm{CH}_{3}\right) 19.97\left(\mathrm{Ph}-\mathrm{CH}_{3}\right)$. IR $\left(\mathrm{CH}_{2} \mathrm{Cl}_{2}\right): v_{\max } 1716,1270,1152$. HRMS calcd for $\mathrm{C}_{13} \mathrm{H}_{17} \mathrm{O}[\mathrm{M}+\mathrm{H}]$ 189.1279, found 189.1289 .

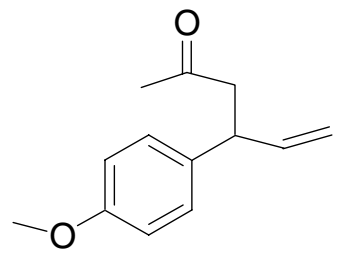

2d: pale yellow oil: ${ }^{1} \mathrm{H}$ NMR $\left(400 \mathrm{MHz}, \mathrm{CDCl}_{3}\right) \delta 7.14(\mathrm{~d}, J=6.5 \mathrm{~Hz}, 2 \mathrm{H}: 2$ arom $\mathrm{H})$, $6.87\left(\mathrm{~d}, J=6.5 \mathrm{~Hz}, 2 \mathrm{H}: 2\right.$ arom $\mathrm{H}$ ), 5.97 (ddd, $J=7 \mathrm{~Hz}, 10.3 \mathrm{~Hz}, 17 \mathrm{~Hz}, 1 \mathrm{H}: \mathrm{CH}=\mathrm{CH}_{2}$ ), $5.06\left(\mathrm{~d}, J=10.3 \mathrm{~Hz}, 1 \mathrm{H}: \mathrm{CH}=\mathrm{CH}(H)_{\mathrm{cis}}\right), 5.02\left(\mathrm{~d}, J=17 \mathrm{~Hz}, 1 \mathrm{H}: \mathrm{CH}=\mathrm{CH}(H)_{\text {trans }}\right) 3.88$ (app. q, $J=7 \mathrm{~Hz}, 1 \mathrm{H}: \mathrm{CH}$ ), 3.80 (s, $\left.3 \mathrm{H}: \mathrm{OCH}_{3}\right) 2.87$ (dd, $J=7 \mathrm{~Hz}, 16 \mathrm{~Hz}, 1 \mathrm{H}$ : diastereotopic $\left.\mathrm{CH}_{2}\right) 2.81\left(\mathrm{dd}, J=7 \mathrm{~Hz}, 16 \mathrm{~Hz}, 1 \mathrm{H}\right.$ : diastereotopic $\left.\mathrm{CH}_{2}\right) 2.10(\mathrm{~s}, 3 \mathrm{H}$ : $\left.\mathrm{CH}_{3}\right) .{ }^{13} \mathrm{C}$ NMR $\left(\mathrm{M} 75 \mathrm{~Hz}, \mathrm{CDCl}_{3}\right) \delta 207.71(\mathrm{C}=\mathrm{O}), 158.64$ (arom. $\left.\mathrm{C}-\mathrm{OCH}_{3}\right), 141.28$ $\left(\mathrm{CH}=\mathrm{CH}_{2}\right), 135.18$ (arom. $\left.\mathrm{C}_{-} \mathrm{CH}_{2}\right) 128.97$ (arom. $\left.\mathrm{CH}\right), 114.67\left(\mathrm{CH}=\mathrm{CH}_{2}\right), 114.39$ (arom. $\mathrm{CH}), 55.65\left(\mathrm{OCH}_{3}\right), 49.55\left(\mathrm{CH}_{2}\right), 44.16(\mathrm{CH}), 31.11\left(\mathrm{CH}_{3}\right)$. IR $\left(\mathrm{CH}_{2} \mathrm{Cl}_{2}\right): v_{\max } 1710$, 1244, 1117, 830. HRMS calcd for $\mathrm{C}_{13} \mathrm{H}_{16} \mathrm{O}_{2}[\mathrm{M}+]$ 204.1150, found 204.1146.

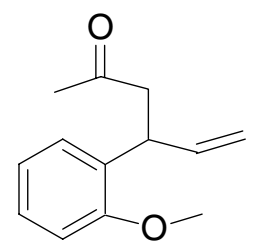

2e: clear oil: ${ }^{1} \mathrm{H}$ NMR $\left(400 \mathrm{MHz}, \mathrm{CDCl}_{3}\right) \delta 7.19(\mathrm{~m}, 2 \mathrm{H}$ : arom $\mathrm{H}), 6.90(\mathrm{~d}, 2 \mathrm{H}: 2$ arom $\mathrm{H}), 6.04$ (ddd, $\left.J=7 \mathrm{~Hz}, 11 \mathrm{~Hz}, 17 \mathrm{~Hz}, 1 \mathrm{H}: \mathrm{CH}=\mathrm{CH}_{2}\right), 5.07(\mathrm{~d}, J=11 \mathrm{~Hz}, 1 \mathrm{H}$ : $\left.\mathrm{CH}=\mathrm{CH}(H)_{\text {cis }}\right), 5.04$ (d, $\left.J=17 \mathrm{~Hz}, 1 \mathrm{H}: \mathrm{CH}=\mathrm{CH}(H)_{\text {trans }}\right) 4.32$ (app. q, $\left.J=7 \mathrm{~Hz}, 1 \mathrm{H}: \mathrm{CH}\right)$, 3.86 (s, $\left.3 \mathrm{H}: \mathrm{OCH}_{3}\right) 2.89$ (dd, $J=7 \mathrm{~Hz}, 16 \mathrm{~Hz}, 1 \mathrm{H}$ : diastereotopic $\left.\mathrm{CH}_{2}\right) 2.83(\mathrm{dd}, J=7$ $\mathrm{Hz}, 16 \mathrm{~Hz}, 1 \mathrm{H}$ : diastereotopic $\left.\mathrm{CH}_{2}\right) 2.14$ (s, 3H: $\left.\mathrm{CH}_{3}\right) .{ }^{13} \mathrm{C} \mathrm{NMR}\left(75 \mathrm{MHz}, \mathrm{CDCl}_{3}\right) \delta$ $208.14(\mathrm{C}=\mathrm{O}), 157.11$ (arom. $\left.\mathrm{C}-\mathrm{OCH}_{3}\right), 140.20\left(\mathrm{CH}=\mathrm{CH}_{2}\right), 131.45$ (arom. $\left.\mathrm{C}-\mathrm{CH}_{2}\right)$ 128.54 (arom. $\mathrm{CH}$ ), 128.06 (arom. $\mathrm{CH}), 121.08$ (arom. $\mathrm{CH}), 115.03\left(\mathrm{CH}=\mathrm{CH}_{2}\right), 111.16$ (arom. CH), $55.79\left(\mathrm{OCH}_{3}\right), 48.57\left(\mathrm{CH}_{2}\right), 38.89(\mathrm{CH}), 30.58\left(\mathrm{CH}_{3}\right)$. IR $\left(\mathrm{CH}_{2} \mathrm{Cl}_{2}\right)$ : v $\max$ 1710, 1234, 1163. HRMS calcd for $\mathrm{C}_{13} \mathrm{H}_{16} \mathrm{O}_{2}[\mathrm{M}+]$ 204.1150, found 204.1159.

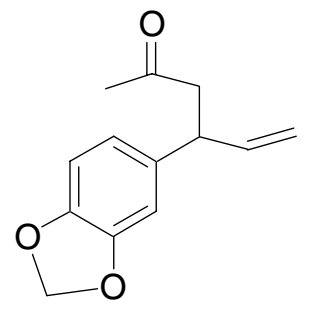

2f: pale yellow oil: ${ }^{1} \mathrm{H}$ NMR $\left(400 \mathrm{MHz}, \mathrm{CDCl}_{3}\right) \delta 6.72(\mathrm{~m}, 3 \mathrm{H}$ : arom $\mathrm{H}$ ), 5.95 (ddd, $J=7$ $\left.\mathrm{Hz}, 10 \mathrm{~Hz}, 17 \mathrm{~Hz}, 1 \mathrm{H}: \mathrm{CH}=\mathrm{CH}_{2}\right), 5.96\left(\mathrm{~s}, 2 \mathrm{H},: \mathrm{O}^{-C_{2}}-\mathrm{O}\right) 5.06(\mathrm{~d}, J=10 \mathrm{~Hz}, 1 \mathrm{H}$ : 
$\left.\mathrm{CH}=\mathrm{CH}(H)_{\text {cis }}\right), 5.02\left(\mathrm{~d}, J=17 \mathrm{~Hz}, 1 \mathrm{H}: \mathrm{CH}=\mathrm{CH}(H)_{\text {trans }}\right), 3.85$ (app. q, $J=7 \mathrm{~Hz}, 1 \mathrm{H}$ : $\mathrm{CH}), 2.85$ (dd, $J=7 \mathrm{~Hz}, 16 \mathrm{~Hz}, 1 \mathrm{H}$ : diastereotopic $\left.\mathrm{CH}_{2}\right) 2.79$ (dd, $J=7 \mathrm{~Hz}, 16 \mathrm{~Hz}, 1 \mathrm{H}$ : diastereotopic $\left.\mathrm{CH}_{2}\right) 2.11$ (s, 3H: $\left.\mathrm{CH}_{3}\right) .{ }^{13} \mathrm{C} \mathrm{NMR}\left(75 \mathrm{MHz}, \mathrm{CDCl}_{3}\right) \delta 207.46(\mathrm{C}=\mathrm{O})$, 148.16 (arom. C), 146.55 (arom. C), $141.04\left(\mathrm{CH}=\mathrm{CH}_{2}\right), 137.05$ (arom. C) 121.00 (arom. $\mathrm{CH}), 114.85\left(\mathrm{O}-\mathrm{CH}_{2}-\mathrm{O}\right), 108.73$ (arom. $\left.\mathrm{CH}\right), 108.43$ (arom. $\left.\mathrm{CH}\right), 101.35\left(\mathrm{CH}=\mathrm{CH}_{2}\right)$, $49.46\left(\mathrm{CH}_{2}\right), 44.58(\mathrm{CH}), 31.12\left(\mathrm{CH}_{3}\right)$. IR $\left(\mathrm{CH}_{2} \mathrm{Cl}_{2}\right): v_{\max } 1710,1152,1040 . \mathrm{HRMS}$ calcd for $\mathrm{C}_{13} \mathrm{H}_{14} \mathrm{O}_{3}[\mathrm{M}+]$ 218.0943, found 218.0926 .

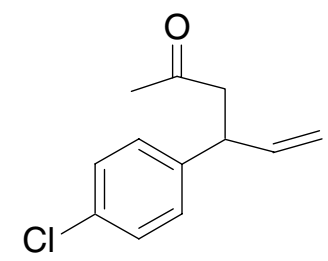

2g: yellow oil: ${ }^{1} \mathrm{H}$ NMR $\left(400 \mathrm{MHz}, \mathrm{CDCl}_{3}\right) \delta 7.28(\mathrm{~d}, J=8 \mathrm{~Hz}, 2 \mathrm{H}: 2$ arom $\mathrm{H}), 7.18(\mathrm{~d}, J$ $=8 \mathrm{~Hz}, 2 \mathrm{H}: 2$ arom H), $5.94\left(\mathrm{ddd}, J=7 \mathrm{~Hz}, 10.3 \mathrm{~Hz}, 17 \mathrm{~Hz}, 1 \mathrm{H}: \mathrm{CH}=\mathrm{CH}_{2}\right), 5.09(\mathrm{~d}, J=$ $\left.10.3 \mathrm{~Hz}, 1 \mathrm{H}: \mathrm{CH}=\mathrm{CH}(H)_{\text {cis }}\right), 5.02\left(\mathrm{~d}, J=17 \mathrm{~Hz}, 1 \mathrm{H}: \mathrm{CH}=\mathrm{CH}(H)_{\text {trans }}\right) 3.92$ (app. q, $J=7$ $\mathrm{Hz}, 1 \mathrm{H}: \mathrm{CH}), 2.89$ (dd, $J=7 \mathrm{~Hz}, 16 \mathrm{~Hz}, 1 \mathrm{H}$ : diastereotopic $\left.\mathrm{CH}_{2}\right) 2.81(\mathrm{dd}, J=7 \mathrm{~Hz}, 16$ $\mathrm{Hz}, 1 \mathrm{H}$ : diastereotopic $\left.\mathrm{CH}_{2}\right) 2.12\left(\mathrm{~s}, 3 \mathrm{H}: \mathrm{CH}_{3}\right) .{ }^{13} \mathrm{C} \mathrm{NMR}\left(75 \mathrm{MHz}, \mathrm{CDCl}_{3}\right) \delta 207.03$ $(\mathrm{C}=\mathrm{O}), 141.67$ (arom C), $140.50\left(\mathrm{CH}=\mathrm{CH}_{2}\right), 132.73$ (arom. C) 129.46 (arom. $\left.\mathrm{CH}\right)$, $129.13(\operatorname{arom~CH}), 115.40\left(\mathrm{CH}=\mathrm{CH}_{2}\right), 49.18\left(\mathrm{CH}_{2}\right), 44.11(\mathrm{CH}), 31.11\left(\mathrm{CH}_{3}\right)$. IR $\left(\mathrm{CH}_{2} \mathrm{Cl}_{2}\right): v_{\max } 1716,1485,1163,1014$. HRMS calcd for $\mathrm{C}_{12} \mathrm{H}_{14} \mathrm{OCl}[\mathrm{M}+\mathrm{H}] 209.0733$, found 209.0728.

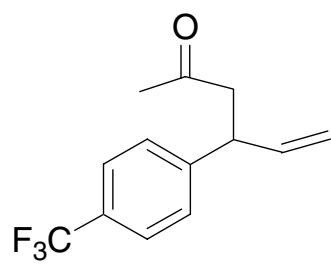

2h: pale yellow oil: ${ }^{1} \mathrm{H}$ NMR (400 MHz, $\left.\mathrm{CDCl}_{3}\right) \delta 7.58(\mathrm{~d}, J=8 \mathrm{~Hz}, 2 \mathrm{H}: 2$ arom $\mathrm{H}$ ), 7.35 (d, $J=8 \mathrm{~Hz}, 2 \mathrm{H}: 2$ arom H), 5.51 (ddd, $J=7 \mathrm{~Hz}, 10.3 \mathrm{~Hz}, 17 \mathrm{~Hz}, 1 \mathrm{H}: \mathrm{CH}=\mathrm{CH}_{2}$ ), 5.12 (d, $\left.J=10.3 \mathrm{~Hz}, 1 \mathrm{H}: \mathrm{CH}=\mathrm{CH}(H)_{\text {cis }}\right), 5.05\left(\mathrm{~d}, J=17 \mathrm{~Hz}, 1 \mathrm{H}: \mathrm{CH}=\mathrm{CH}(H)_{\text {trans }}\right) 4.02$ (app. q, $J=7 \mathrm{~Hz}, 1 \mathrm{H}: \mathrm{CH}), 2.94$ (dd, $J=7 \mathrm{~Hz}, 17 \mathrm{~Hz}, 1 \mathrm{H}$ : diastereotopic $\left.\mathrm{CH}_{2}\right) 2.86(\mathrm{dd}, J=7$ $\mathrm{Hz}, 17 \mathrm{~Hz}, 1 \mathrm{H}$ : diastereotopic $\left.\mathrm{CH}_{2}\right) 2.13\left(\mathrm{~s}, 3 \mathrm{H}: \mathrm{CH}_{3}\right) .{ }^{13} \mathrm{C} \mathrm{NMR}\left(75 \mathrm{MHz}, \mathrm{CDCl}_{3}\right) \delta$ $206.67(\mathrm{C}=\mathrm{O}), 147.33(\operatorname{arom} C-\mathrm{CH}), 140.07\left(\mathrm{CH}=\mathrm{CH}_{2}\right), 129.28\left(\operatorname{arom} C-\mathrm{CF}_{3}, \mathrm{q}, J_{\mathrm{CF}}=54\right.$ $\mathrm{Hz}), 128.47$ (arom. $\mathrm{CH}), 125.94\left(\mathrm{CF}_{3}, \mathrm{q}, J_{\mathrm{CF}}=4 \mathrm{~Hz}\right), 115.84\left(\mathrm{CH}=\mathrm{CH}_{2}\right), 48.98\left(\mathrm{CH}_{2}\right)$, $44.45(\mathrm{CH}), 31.06\left(\mathrm{CH}_{3}\right)$. IR $\left(\mathrm{CH}_{2} \mathrm{Cl}_{2}\right): v_{\max } 1716,1613,1326,1168$. HRMS calcd for $\mathrm{C}_{13} \mathrm{H}_{12} \mathrm{~F}_{3} \mathrm{O}[\mathrm{M}-\mathrm{H}]$ 241.0840, found 241.0840. 


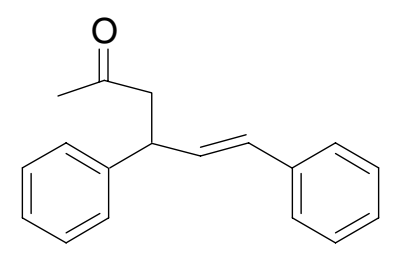

2j: ${ }^{6}$ yellow oil: ${ }^{1} \mathrm{H}$ NMR $\left(400 \mathrm{MHz}, \mathrm{CDCl}_{3}\right) \delta 7.30(\mathrm{~m}, 10 \mathrm{H}$ : arom $\mathrm{H}), 6.38(\mathrm{~m}, 2 \mathrm{H}$ : ambiguous $\mathrm{CH}=$ ), 4.12 (app. q, $J=7 \mathrm{~Hz}, 1 \mathrm{H}$ : $\mathrm{CH}$ ), 3.01 (dd, $J=7 \mathrm{~Hz}, 16 \mathrm{~Hz}, 1 \mathrm{H}$ : diastereotopic $\left.\mathrm{CH}_{2}\right) 2.95\left(\mathrm{dd}, J=7 \mathrm{~Hz}, 16 \mathrm{~Hz}, 1 \mathrm{H}\right.$ : diastereotopic $\left.\mathrm{CH}_{2}\right), 2.14(\mathrm{~s}, 3 \mathrm{H}$ : $\left.\mathrm{CH}_{3}\right) .{ }^{13} \mathrm{C} \mathrm{NMR}\left(75 \mathrm{MHz}, \mathrm{CDCl}_{3}\right) \delta 207.36(\mathrm{C}=\mathrm{O}), 143.35$ (arom. C), 137.49 (arom. C), $132.76(\mathrm{CH}=), 130.39(\mathrm{CH}=), 129.13$ (arom. $\mathrm{CH}), 128.91$ (arom. $\mathrm{CH}), 128.06$ (arom. $\mathrm{CH}), 127.75$ (arom. $\mathrm{CH}), 127.12$ (arom. $\mathrm{CH}), 126.65$ (arom. $\mathrm{CH}), 49.82\left(\mathrm{CH}_{2}\right), 44.36$ $(\mathrm{CH}), 31.20\left(\mathrm{CH}_{3}\right)$. IR $\left(\mathrm{CH}_{2} \mathrm{Cl}_{2}\right): v_{\max } 1710,1490,1255$. HRMS calcd for $\mathrm{C}_{18} \mathrm{H}_{18} \mathrm{O}[\mathrm{M}+]$ 250.1358 , found 250.1352 .

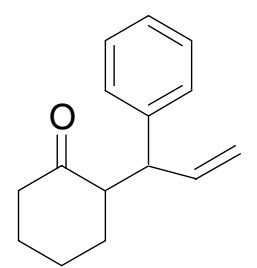

5: yellow oil: ${ }^{1} \mathrm{H}$ NMR $\left(400 \mathrm{MHz}, \mathrm{CDCl}_{3}\right)$ Major Diastereomer: $\delta 7.27(\mathrm{~m}, 5 \mathrm{H}$ : arom $\mathrm{H}$ ), 6.07 (ddd, $\left.J=7 \mathrm{~Hz}, 10 \mathrm{~Hz}, 17 \mathrm{~Hz}, 1 \mathrm{H}: \mathrm{C} H=\mathrm{CH}_{2}\right), 5.06$ (m, $\left.2 \mathrm{H}: \mathrm{CH}=\mathrm{CH}_{2}\right), 3.75(\mathrm{t}, J=9$ $\mathrm{Hz}, 1 \mathrm{H}: \quad \mathrm{CH}-\mathrm{CH}=\mathrm{CH}_{2}$ ), 2.82 (m, 1H: $\mathrm{COCH}$ ) 2.46-1.24 (broad m, overlapping cyclohexyl $\mathrm{CH}_{2}$ 's). Minor Diastereomer: $\delta 7.27(\mathrm{~m}, 5 \mathrm{H}$ : arom H), 5.99 (ddd, $J=7 \mathrm{~Hz}, 10$ $\mathrm{Hz}, 17 \mathrm{~Hz}, 1 \mathrm{H}: \mathrm{CH}=\mathrm{CH}_{2}$ ), 5.06 (m, 2H: $\left.\mathrm{CH}=\mathrm{CH}_{2}\right), 3.83$ (t, $J=9 \mathrm{~Hz}, 1 \mathrm{H}: \mathrm{CH}-\mathrm{CH}=\mathrm{CH}_{2}$ ), 2.82 (m, 1H: $\mathrm{COCH}) 1.84$ (broad m, overlapping cyclohexyl $\mathrm{CH}_{2}$ 's). ${ }^{13} \mathrm{C}$ NMR (75 $\mathrm{MHz}, \mathrm{CDCl}_{3}$ ) Major and Minor Diastereomer: $\delta$ 213.15; $212.17(\mathrm{C}=\mathrm{O}), 143.67 ; 142.07$ (arom. C), 140.52; $139.64\left(\mathrm{CH}=\mathrm{CH}_{2}\right), 128.98 ; 128.85$ (arom. $\left.\mathrm{CH}\right), 128.24$ (arom. $\mathrm{CH}$ ), 126.94; 126.66 (arom. $\mathrm{CH}), 116.65 ; 115.34\left(\mathrm{CH}=\mathrm{CH}_{2}\right), 55.80 ; 55.65(\mathrm{COCH}), 49.76$; $49.44\left(\mathrm{CH}-\mathrm{CH}=\mathrm{CH}_{2}\right), 42.76 ; 42.51\left(\mathrm{CH}_{2}\right), 32.35 ; 32.03\left(\mathrm{CH}_{2}\right), 28.96 ; 28.80\left(\mathrm{CH}_{2}\right)$, 24.80; $24.14\left(\mathrm{CH}_{2}\right)$. IR $\left(\mathrm{CH}_{2} \mathrm{Cl}_{2}\right): v_{\max } 1705,1445,1214$. HRMS calcd for $\mathrm{C}_{15} \mathrm{H}_{19} \mathrm{O}$ $[\mathrm{M}+\mathrm{H}] 215.1436$, found 215.1439.

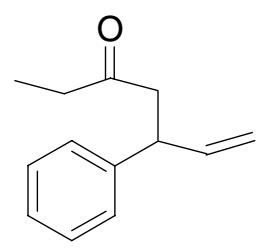

9a: pale yellow oil: ${ }^{1} \mathrm{H}$ NMR (400 MHz, $\left.\mathrm{CDCl}_{3}\right) \delta 7.28(\mathrm{~m}, 5 \mathrm{H}$ : arom $\mathrm{H}$ ), 6.00 (ddd, $J=$ $\left.7 \mathrm{~Hz}, 10 \mathrm{~Hz}, 17 \mathrm{~Hz}, 1 \mathrm{H}: \mathrm{CH}=\mathrm{CH}_{2}\right), 5.08\left(\mathrm{~d}, J=10 \mathrm{~Hz}, 1 \mathrm{H}: \mathrm{CH}=\mathrm{CH}(H)_{\mathrm{cis}}\right), 5.04(\mathrm{~d}, J=$

${ }^{6}$ Oi, S.; Honma, Y.; Inoue, Y. Org. Lett. 2002, 4, 667-669. 
$\left.17 \mathrm{~Hz}, 1 \mathrm{H}: \mathrm{CH}=\mathrm{CH}(H)_{\text {trans }}\right) 3.95$ (app. q, $\left.J=7 \mathrm{~Hz}, 1 \mathrm{H}: \mathrm{CH}\right), 2.89$ (dd, $J=7 \mathrm{~Hz}, 16 \mathrm{~Hz}$, 1H: diastereotopic $\left.\mathrm{CH}-\mathrm{CH}_{2}\right) 2.82\left(\mathrm{dd}, \mathrm{J}=7 \mathrm{~Hz}, 16 \mathrm{~Hz}, 1 \mathrm{H}\right.$ : diastereotopic $\left.\mathrm{CH}-\mathrm{CH}_{2}\right) 2.41$ (dq, $J=7 \mathrm{~Hz}, 10 \mathrm{~Hz}, 1 \mathrm{H}$ : diastereotopic $\left.\mathrm{CH}_{2}-\mathrm{CH}_{3}\right) 2.33(\mathrm{dq}, J=7 \mathrm{~Hz}, 10 \mathrm{~Hz}, 1 \mathrm{H}$ : diastereotopic $\left.\mathrm{CH}_{2}-\mathrm{CH}_{3}\right) 1.01\left(\mathrm{t}, J=7 \mathrm{~Hz}, 3 \mathrm{H}: \mathrm{CH}_{3}\right) .{ }^{13} \mathrm{C} \mathrm{NMR}\left(75 \mathrm{MHz}, \mathrm{CDCl}_{3}\right) \delta$ $210.10(\mathrm{C}=\mathrm{O}), 143.36$ (arom. C), $141.08\left(\mathrm{CH}=\mathrm{CH}_{2}\right), 129.01$ (arom. $\left.\mathrm{CH}\right), 128.03$ (arom. $\mathrm{CH}), 126.98$ (arom. $\mathrm{CH}), 114.97\left(\mathrm{CH}=\mathrm{CH}_{2}\right), 48.22\left(\mathrm{CH}-\mathrm{CH}_{2}\right), 45.00(\mathrm{CH}), 37.185\left(\mathrm{CH}_{2}-\right.$ $\left.\mathrm{CH}_{3}\right) 7.97\left(\mathrm{CH}_{3}\right)$. IR $\left(\mathrm{CH}_{2} \mathrm{Cl}_{2}\right): v_{\max } 1716,1449,1106$. HRMS calcd for $\mathrm{C}_{13} \mathrm{H}_{17} \mathrm{O}$ $[\mathrm{M}+\mathrm{H}]$ 189.1279, found 189.1276. ${ }^{1} \mathrm{H}$ NMR spectroscopy of the crude mixture confirms that no 3-methyl-4-phenyl-5-hexene-2-one was produced. ${ }^{7}$

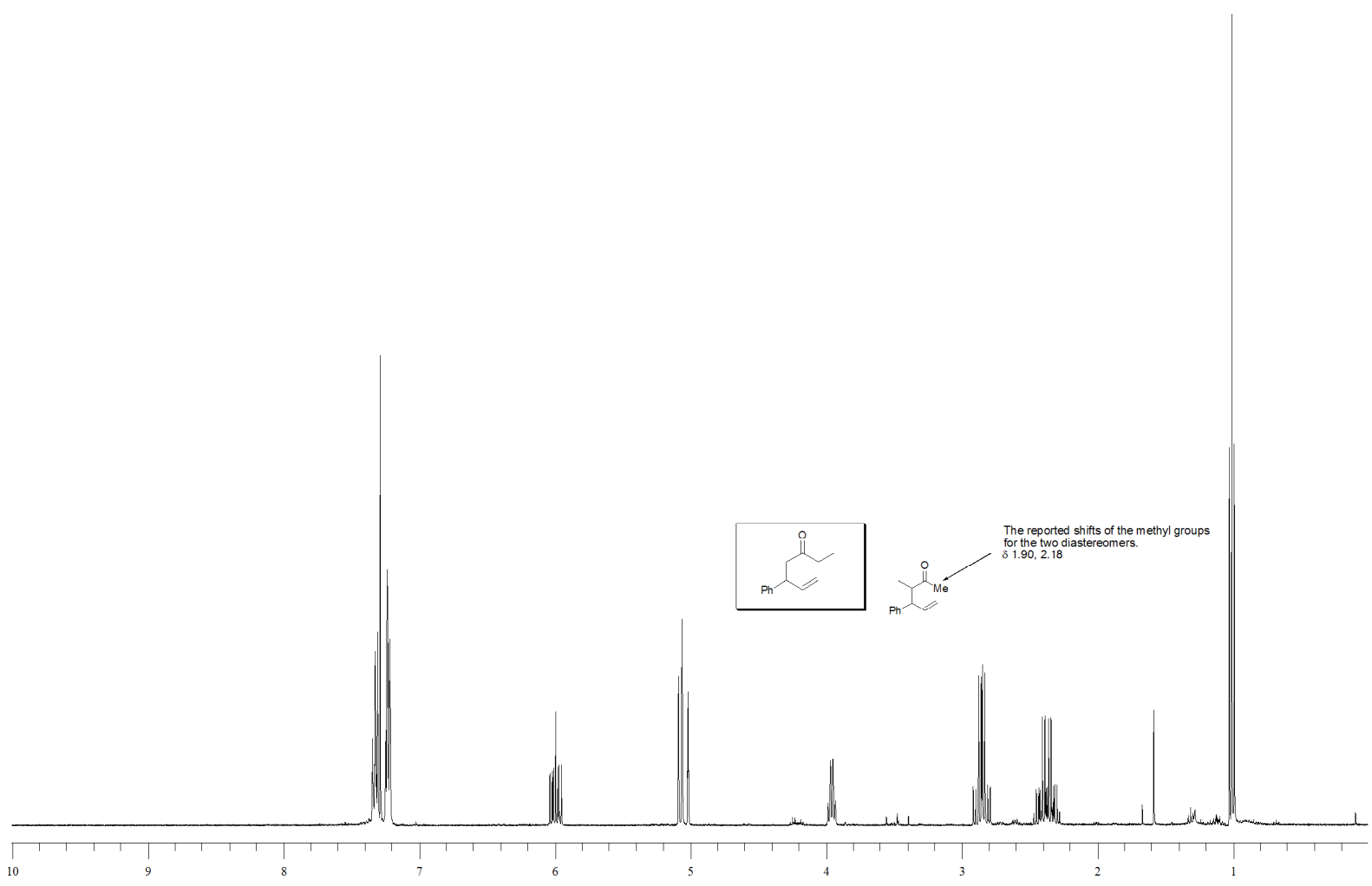

Figure 1. ${ }^{1} \mathrm{H}$ NMR spectrum of the crude mixture containing 9a. The reported chemical shifts for the methyl ketone groups of the two diastereomers of 3-methyl-4-phenyl-5-hexene-2-one are $\delta 1.90$ and 2.18. The absence of these peaks confirms that the enolate generated did not rearrange.

Crossover Experiment: An NMR tube was charged with approximately equal quantities of $8 \mathbf{a}$ and 1g. Bipyridine $(10 \mathrm{~mol} \%)$ and $\left[\mathrm{Cp}^{*} \mathrm{RuCl}\right]_{4}(2.5 \mathrm{~mol} \%)$ were added, followed by $\mathrm{CD}_{2} \mathrm{Cl}_{2}(550 \mu \mathrm{L})$. Upon reaction completion the mixture was analyzed by $\mathrm{GC}$ and MS. ${ }^{1} \mathrm{H}$ NMR spectroscopy of the mixture confirms that no 3-methyl-4-phenyl-5hexene-2-one was produced. ${ }^{7}$

\footnotetext{
${ }^{7}$ Daub, G. W.; McCoy, M. A.; Sanchez, M. G.; Carter, J. S. J. Org. Chem. 1983, 48, 3876-83.
} 
S-7

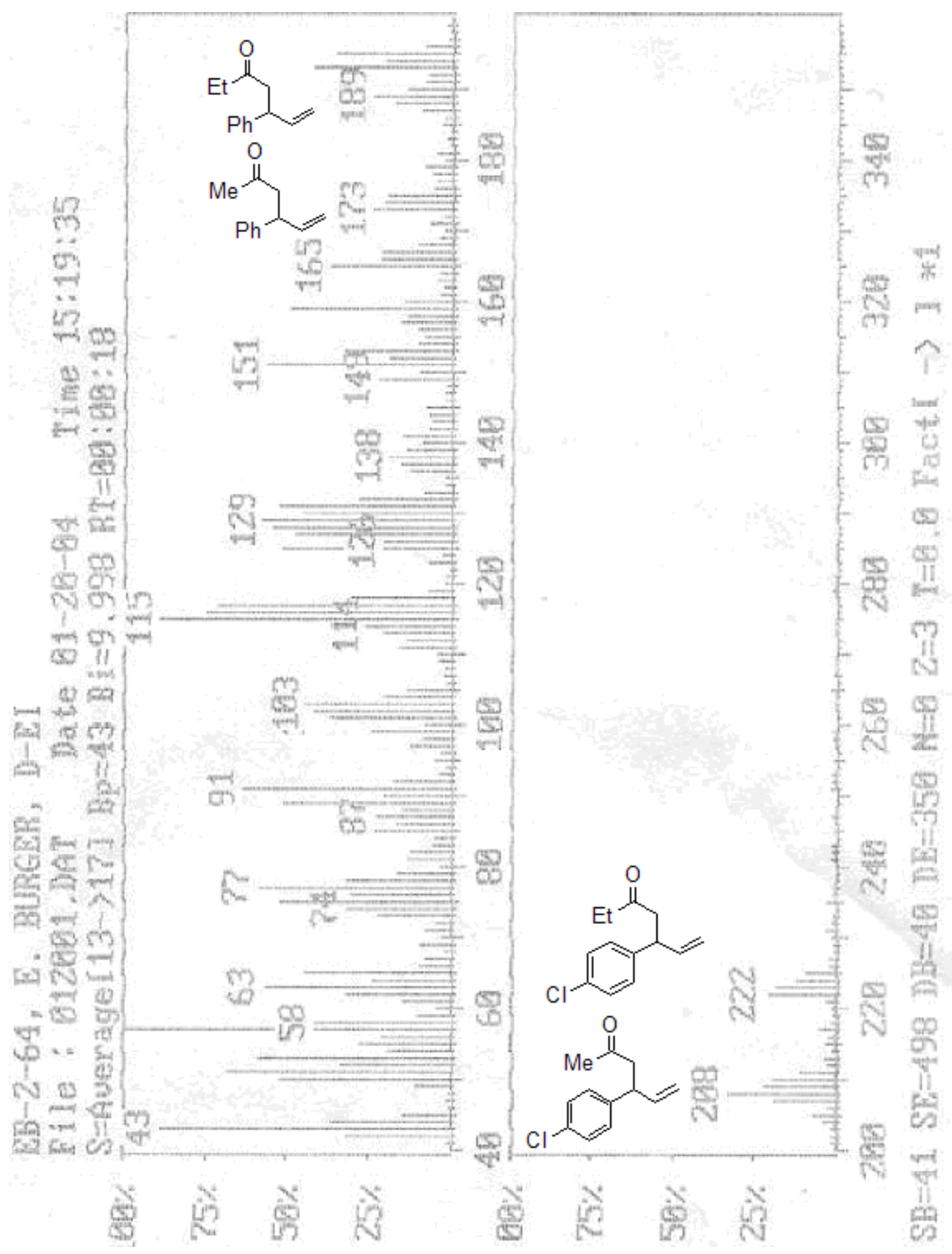

